\title{
MOLECULAR GAS IN THE POSTSTARBURST GALAXY NGC7331
}

T. TOSAKI

Gunma Astronomical Observatory

Ohdomo, 1-18-7, Maebashi, Gunma, 371, Japan

AND

Y. SHIOYA

Astronomical Institute, Tohoku University

Aoba, Sendai, 980-77, Japan

\section{Introduction}

To understand the origin and evolution of starburst activity, we must study the full evolution of starburst; i.e., pre-, on-going, and post-starburst phases. It seems reasonable to suppose the numerous A-type stars indicate past starburst and they show strong Balmer absorption. NGC7331, nearby early-type spiral galaxy, is one of the poststarburst galaxies which show strong Balmer absorption. The optical spectra of NGC7331 were dominated by component of intermediate-age $\left(5 \times 10^{9}\right.$ years $)$ stellar populations (Ohyama \& Taniguchi 1996). We present the result of the high resolution CO observations of NGC7331 using Nobeyama Milimeter Array.

\section{Results and Discussion}

The molecular gas is distributed in two bow-like structures, which seem to be parts of the ring-like structure seen in the radio continuum. The ring has a radius of $\sim 3 \mathrm{kpc}$, corresponding to the turn-over radius of the rotation curves obtained both by radio and optical observations (Marcelin et al. 1994; Nishiyama 1995). In the ring-like structure, the observed azimuthal averaged gas mass surface density, $100-200 M_{\odot} \mathrm{pc}^{-2}$. We found that the ring consists of several clumps with a typical size and mass of $10^{\prime \prime}(690$ pc) and $5 \times 10^{7} M_{\odot}$, respectively. On the other hand, we could not found 
Figure 1. Total integrated intensity map was superposed on the $20 \mathrm{~cm}$ image (Cowan et al. 1994). The synthesized beam is revealed in the bottom left of the figure. The contour interval is $4.1 \mathrm{Jy} \mathrm{km} \mathrm{s}^{-1}$ beam $^{-1}$, corresponding to $1 \sigma$ and the lowest contour is $2 \sigma$. The cross indicates the position of galactic nucleus determined from peak of radio continuum. The field of view is represented by circle.

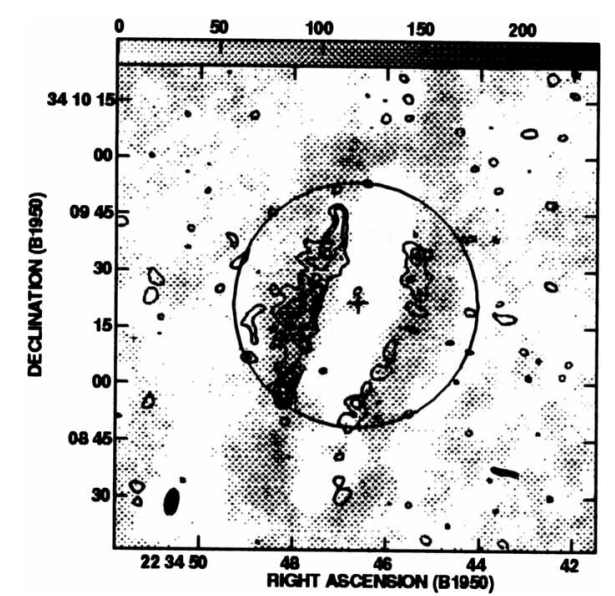

CO emission in the central region (the upper limit of gas mass surface density $\sim 60 M_{\odot} \mathrm{pc}^{-2}$ ), showing the poststarburst feature. These features are different from those of the other poststarburst galaxies, NGC4736 and NGC5055, which show the central concentrations of molecular gas (Kuno et al. 1997; Nishiyama 1995).

The Toomre's $Q$ values, the ratio of critical density at which the disk is unstable and breaks up to surface density, are $0.2-0.3$ in the ring (cf. Kennicutt 1989). On the other hand, the upper limit of the surface density in the central region including poststarburst region is significantly smaller than the critical density, and thus the $Q$ values in the central region exceed unity if we took consideration of the problem for a missing flux. Therefore, the molecular gas in the ring is unstable and that in the central region is stable if there were gas disk. This indicates that the activity of star formation in NGC7331 seems to be related to the stability of gas disk. However, the distribution of mass surface density in NGC7331 is different from that of the critical surface density, and this suggests that the global molecular gas distribution in NGC7331 are not controlled by the disk stability. We proposed the possible scenario that the central hole of molecular gas in NGC7331 were due to the gas consumption over the long evolution after the past starburst event, e.g., continuous slight star formation or accretion toward nucleus.

\section{References}

Cowan, J.J., Romanishin, W. \& Branch, D. 1994, ApJ, 436, L139

Kennicutt, R.C., 1989, ApJ, 344, 685

Kuno, N., Tosaki, T., Nishiyama, K. \& Nakai, N., 1997, PASJ, 49, 275

Marcelin, M., Petrosin, A. R., Amram, A. R. \& Boulesteix, J., 1994, A\&A, 282, 363

Nishiyama, K. 1995, PhD thesis, the Graduate University of Advanced Studies

Ohyama, Y. \& Taniguchi, Y., 1996, in The Physics of Liners in View of Recent Observations, (eds.) E. Eracleous, A. Koratkar, C. Leitherer, and L. Ho, ASP Conf.Ser.Vol.103, p.205 